\title{
A THEOREM ON PARTIALLY ORDERED SETS OF ORDER-PRESERVING MAPPINGS
}

\author{
DWIGHT DUFFUS AND RUDOLF WILLE ${ }^{1}$
}

\begin{abstract}
Let $P$ be a partially ordered set and let $P^{P}$ denote the set of all order-preserving mappings of $P$ to $P$ ordered by $f<g$ in $P^{P}$ if $f(p)<g(p)$ for all $p \in P$. We prove that if $P$ and $Q$ are finite, connected partially ordered sets and $P^{P} \simeq Q^{Q}$ then $P \simeq Q$.
\end{abstract}

Is a partially ordered set determined by its order-preserving mappings? $\mathrm{L}$. M. Gluskin [4] has shown that the set of order-preserving mappings of a partially ordered set $P$ as a semigroup of transformations determines $P$ up to duality. Let us consider the set $P^{P}$ of order-preserving mappings of $P$ as $a$ partially ordered set: $f \leqslant g$ in $P^{P}$ if $f(p) \leqslant g(p)$ for all $p \in P$. (If $P$ is a finite lattice then $P$ is determined by $P^{P}[6]$.)

The purpose of this note is to establish the following.

TheOREM. Let $P$ and $Q$ be finite, connected partially ordered sets. Then $P^{P} \cong Q^{Q}$ implies $P \cong Q$.

In proving the theorem, we first change the problem from one concerning exponents $X^{Y}$ of partially ordered sets to one about products $X Y$ (or $X \times Y$ ) of partially ordered sets. The change is accomplished with a description of the covering relation in an exponent.

Recall that for elements $a>b$ in a partially ordered set $X$, a covers $b$ if $a \geqslant c>b$ implies $a=c$. Let $V(X)$ denote the set of elements of $X$ with a unique lower cover in $X$. Let $X^{d}$ denote the dual of $X$. The following result is due to D. Duffus and I. Rival [3].

Logarithmic property. Let $X$ and $Y$ be finite partially ordered sets. If $X$ has a least element, then $V\left(X^{Y}\right) \cong V(X) \times Y^{d}$.

Let $X$ be a finite partially ordered set. For $x \in X$ we let $[x)=\{y \in X \mid y$ $\geqslant x\}$ and let $V(x)=V([x)$ ). We also let the length $l(X)$ of $X$ be defined by

$$
l(X)=\sup \{|C|-1 \mid C \subseteq X, C \text { is a chain }\}
$$

and the depth $\delta(x)$ of $x$ in $X$ is given by

$$
\delta(x)=\sup \{l(C) \mid C \subseteq X, C \text { is a chain and } \inf (C)=x\} .
$$

It is clear that $x$ has maximum depth in $X$ if and only if $\delta(x)=l(X)$.

Received by the editors February 16, 1978.

AMS (MOS) subject classifications (1970). Primary 06A10.

${ }^{1}$ The work presented here was supported in part by the National Research Council of Canada. 
Let $P$ and $Q$ be finite, connected partially ordered sets and let $\varphi$ be an isomorphism of $P^{P}$ onto $Q^{Q}$. Let $f \in P^{P}$ satisfy $\delta(f)=l\left(P^{P}\right)$. Since $l\left(X^{Y}\right)=$ $l(X) \cdot|Y|$ for finite partially ordered sets $X$ and $Y[3], \delta(f)=l(P) \cdot|P|$. Moreover,

$$
\delta(f) \leqslant \sum_{a \in P} \delta(f(a)) ;
$$

therefore, $\delta(f(a))=l(P)$ for all $a \in P$. As $f(P)$ is connected, we conclude that $f$ is a constant mapping, say $f(P)=\{a\}$, where $\delta(a)=l(P)$. Similarly, if $\varphi(f)=g$ then $g$ is an element of maximum depth in $Q^{Q}$ and $g(Q)=\{b\}$, where $\delta(b)=l(Q)$. It is not difficult to see that

$$
[a)^{P} \cong[f) \cong[g) \cong[b)^{Q} .
$$

We apply the logarithmic property to (1) and obtain

$$
V(a) \times P^{d} \cong V(b) \times Q^{d} .
$$

Let us apply an extension of J. Hashimoto's common refinement theorem for product factorizations to (2) [5] (cf. [2]). We obtain partially ordered sets $R, S, T$, and $U$ satisfying

$$
V(a) \cong R S^{d}, \quad P \cong T U, \quad V(b) \cong R T^{d}, \quad Q \cong S U .
$$

Let $a \in P$ correspond to $(t, u) \in T U$ and $b \in Q$ correspond $\left(s, u^{\prime}\right) \in S U$ under the isomorphisms in (3). Observe that $(x, y) \in X Y$ satisfies $(x, y) \in$ $V(X Y)$ if and only if $x \in V(X)$ and $y$ is a minimal element of $Y$ or $x$ is minimal in $X$ and $y \in V(Y)$. Then $[a) \cong[t] \times[u)$ and $[b] \cong[s] \times\left[u^{\prime}\right)$; hence,

$$
R S^{d} \cong V(a) \cong V(t)+V(u)
$$

and

$$
R T^{d} \cong V(b) \cong V(s)+V\left(u^{\prime}\right) .
$$

(We use $X+Y$ to denote the disjoint union of partially ordered sets $X$ and $Y$ [1].) $S$ and $T$ are connected partially ordered sets; therefore, $R \cong R_{1}+R_{2} \cong$ $R_{3}+R_{4}$ and $V(t) \cong R_{1} S^{d}, V(s) \cong R_{3} T^{d}$ [1]. It now follows that

$$
|V(t)|=\left|R_{1}\right||S| \geqslant\left|R_{1}\right||V(s)|=\left|R_{1}\right|\left|R_{3}\right||T| .
$$

Since $t \notin V(t), V(t)$ is a proper subset of $T$. Therefore, $R_{1}=\varnothing$ or $R_{3}=\varnothing$. Let us suppose that $R_{1}=\varnothing$. Then $V(t)=\varnothing$ and, because $t$ is a minimal element of the connected partially ordered set $T,|T|=1$. Hence, from (3),

$$
U^{U} \cong P^{P} \cong Q^{Q} \cong\left(S^{S U}\right)\left(U^{U}\right)^{S} .
$$

It follows that $|S|=1$ and that $P \cong U \cong Q$. In the case that $R_{3}=\varnothing$, a similar argument establishes that $P \cong Q$.

The proof of the theorem is now complete.

We observe that the same argument as used in the proof of the Theorem verifies a curious "cancellation" law. 
COROllary. Let $P$ and $Q$ be finite, connected partially ordered sets. Then $P Q \simeq Q^{P}$ implies $P \cong Q$.

The corollary does not hold for all finite antichains $n: 2^{4} \cong 4^{2}$ !

\section{REFERENCES}

1. G. Birkhoff, Extended arithmetic, Duke Math. J. 3 (1937), 311-316.

2. D. Duffus, Toward a theory of finite partially ordered sets, $\mathrm{Ph} . \mathrm{D}$. Thesis, University of Calgary, 1978.

3. D. Duffus and I. Rival, A logarithmic property for exponents of partially ordered sets, Canad. J. Math. 30 (1978), 797-807.

4. L. M. Gluskin, Semigroups of isotone transformations, Uspehi Mat. Nauk 16 (1961), 157-162. MR 24 \# A1336.

5. J. Hashimoto, On direct product decomposition of partially ordered sets, Ann. of Math. (2) 54 (1951), 315-318. MR 13, 201.

6. R. Wille, Cancellation and refinement results for function lattices, Houston J. Math. (to appear).

Department of Mathematics, University of Calgary, Calgary, Canada

Department of Mathematics, Technische Hochschule Darmstadt, Darmstadt, Federal RePublic OF GeRMany 